\title{
Montée aux médias sur fond de scandale : Le cas de Soumission de Michel Houellebecq
}

\author{
Ugo Ruiz et Mette Tjell (Dép. de Langues er Littératures - Université \\ de Gothenburg, Suède)
}

\begin{abstract}
The article questions the dynamics of the contemporary literary scandal through the controversy caused by the novel Submission (2015) by the French writer Michel Houellebecq. This novel seeks to provoke the reader by staging a scenario that plays on the fears of Islam. The study focuses on a TV interview with Houellebecq at the Journal de France 2, on January 6, 2015, the day before the publication of the novel. The analyse shows that the journalist aims to bring out the writer's personal point of view, which is likely to enhance the desirable polemic, whereas the writer remains evasive asserting the autonomy of literature. From this performance on the media scene, one can observe the constructed character of literary polemics of today, where the transgression becomes the pretext to attract public attention. This new logic of literary polemics seriously questions the possibility of real scandals to arise in literature.
\end{abstract}

Keywords: Michel Houellebecq, literary scandal, transgression, mass media, marketing strategies

\section{Introduction}

Nombreuses sont les œuvres qui ont fait date par le scandale qu'ils ont causé, ayant heurté la bienséance ou la morale de leur époque. Parfois ils ont donné lieu à des procès retentissants, comme c'est le cas des Fleurs du mal de Baudelaire et de Madame Bovary de Flaubert, ou encore L'Écume des jours de Boris Vian. Mais hormis témoigner des préoccupations d'une époque donnée, ces scandales, entre bien d'autres, sont autant de preuves que la littérature est restée, malgré sa dite autonomie, soumise à un dispositif de contrôle et de restriction de la langue qui vient autant du public et des médias que du pouvoir politique et juridique. Pourtant, il nous semble que le scandale littéraire aujourd'hui se fait rare. S'il y a régulièrement des polémiques dans le milieu littéraire, peu d'entre elles sont récupérées par les médias de masse et diffusées à un public plus large donnant lieu au scandale. Quand cela arrive, celui-ci ne fait pas long feu et se dissout rapidement dans le flux d'informations des médias. Doit-on en conclure que la littérature est désormais une zone d'expression totalement libre ? Il est permis d'en douter. L'explication n'est-elle pas plutôt à chercher dans la nouvelle dynamique des médias de masse ?

Nous interrogerons le scandale contemporain à partir du cas spécifique de Soumission (2015) de Michel Houellebecq, roman qui a été présenté comme particulièrement polémique lors de sa parution. Il a par la suite suscité de nombreux commentaires dans les médias, d'autant que sa parution a coïncidé avec l'attaque meurtrière de Charlie Hebdo le 7 janvier 
2015, circonstance qui a augmenté la médiatisation du roman. Cet accueil semble ainsi à première vue contenir des éléments propres à un scandale.

Dans le cadre de cette étude, nous tenterons de faire apparaître les enjeux et les tensions au cœur de cet événement littéraire. Dans une première partie théorique, nous proposerons une définition du scandale et de ses spécificités dans le domaine littéraire. Nous essayerons ensuite, dans une deuxième partie, de déterminer ce qui peut être considéré comme provocateur dans le roman. Puis, dans une troisième partie, nous analyserons l'apparition de l'écrivain au Journal de vingt heures de France 2, une interview qui a été donnée juste avant les attentats et qui n'a donc pas été influencée par ces événements.

\section{Paradoxe du scandale littéraire}

Afin de mieux cerner les mécanismes relatifs à la réception de Soumission, il est utile de déterminer d'abord quelques caractéristiques générales des scandales et plus particulièrement des scandales littéraires. Dans l'ouvrage On scandal. Moral Disturbances in Society, Politics and Art (2008), le chercheur américain Ari Adut propose, à partir d'une approche à la fois sociologique et psychologique, des éléments théoriques pour l'étude de scandales. Dans sa définition du scandale, la transgression, qui le plus souvent touche à la sexualité ou à la corruption, occupe une place centrale, et doit en outre éveiller des réactions unanimes chez un large public. En ceci, le scandale diffère de la polémique qui a pour élément moteur une divergence de points de vue, souvent entre spécialistes ${ }^{1}$. Marc Angenot (1982, 34) insiste également sur ce point: marquée par l'opposition des discours, la polémique «suppose un contre-discours antagoniste [...] lequel vise dès lors une double stratégie : démonstration de la thèse et réfutation-disqualification d'une thèse adverse ». En ceci, la polémique se définit surtout comme une modalité parmi d'autres dans le domaine de la rhétorique argumentative (Amossy 2014, 70).

Si l'une des polémiques littéraires les plus connues est sans doute la querelle sur le style de Flaubert, on peut aussi mentionner, en l'occurrence, celle sur le style de Houellebecq, qui a été sujet d'une polémique, entre 1998 et 2010, car jugé par une partie de la critique trop «plat» pour être considéré comme littéraire (Estier 2015). Avec Soumission, comme nous allons le voir, les réactions du public à la dimension politique du roman dépassent de loin le cercle des critiques littéraires, raison pour laquelle la réception de ce dernier fait plus penser à un scandale. Comme le souligne aussi Adut dans sa définition du phénomène, ce n'est d'ailleurs pas tant l'acte transgressif en lui-même que le bruit qu'il engendre qui est important dans l'apparition d'un scandale :

A scandal is an event of varying duration that starts with the publicization of a real, apparent, or alleged transgression to a negatively oriented audience and lasts as long as there is significant and sustained public interest in it. (Adut 2008, 11)

En effet, la perception et les conséquences de la limite franchie dépendent avant tout de l'intérêt des différents acteurs. À partir de cette approche, Adut distingue trois composantes principales dans le scandale : (1) une transgression, (2) quelqu'un qui la rend

\footnotetext{
${ }^{1}$ Citons à ce propos Gilles Philippe qui au début de son ouvrage analysant la querelle sur le style de Flaubert définit ainsi la polémique : «Faut-il le rappeler ? Les polémiques littéraires (comme les autres sans doute) n'ont pas pour vocation première de faire avancer la réflexion, de produire du savoir, de préciser les théories, mais tout simplement de reconfigurer la communauté des gens de lettres ». (Philippe 2004, 8)
} 
publique et (3) un public intéressé (ibid., 12). Dans le cas de la littérature, on peut visualiser ces composantes par le schéma ci-dessous (Fig 1) : l'auteur de la transgression (l'écrivain) ou un tiers soumet un texte à un éditeur qui fait le choix de le publier et de le promouvoir sous forme de livre, s'adressant à des lecteurs potentiels. Le scandale prend finalement forme avec les réactions des médias et/ou de l'autorité.

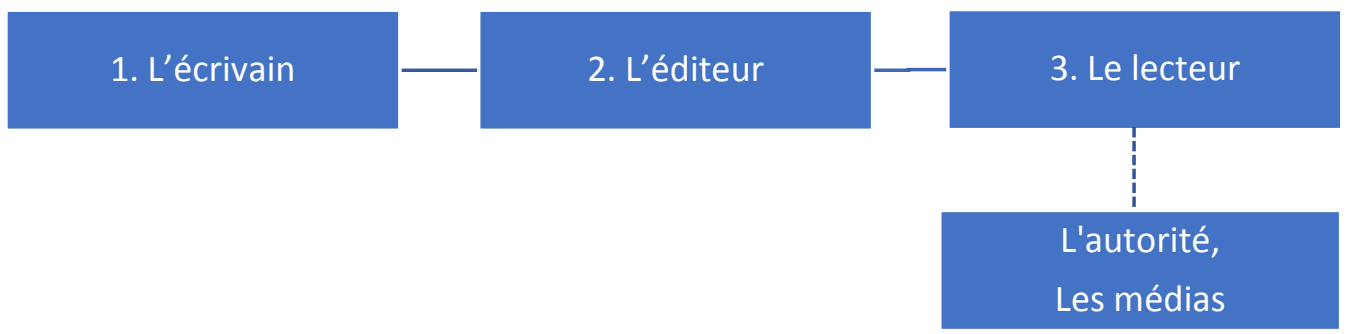

Fig. 1 Scandale littéraire dans sa forme traditionnelle

Si cet enchaînement d'actions peut décrire la montée d'un scandale, l'analyse doit aussi déterminer la nature et la cible de la transgression, qui sont décisives pour comprendre les effets que cette dernière peut entraîner. Selon Adut, une caractéristique définitoire du scandale est notamment que la transgression arrive soit (1) à atteindre de manière négative la réputation d'un individu, d'un groupe ou d'une institution de haut statut, soit (2) à défier le public ou les autorités (ibid., 22), ou bien les deux à la fois. En considérant le fonctionnement propre de la sphère esthétique depuis un siècle en France et en Europe, on peut affirmer que les scandales littéraires correspondent pour la majorité au deuxième type, ayant trait à une provocation. De là, il est, nous semble-t-il, possible d'esquisser deux particularités, quelque peu paradoxales, qui distinguent les scandales en littérature :

I. Même lorsque la transgression atteint le degré de scandale, elle a presque systématiquement un effet positif sur l'image d'auteur, dans la mesure où la transgression des codes esthétiques ou moraux dans ses textes peut être un moyen pour l'écrivain d'affirmer une singularité.

II. Il s'en suit que c'est généralement l'écrivain lui-même qui cherche à rendre publique la transgression par laquelle il peut susciter un intérêt du public. Cette mise en spectacle, qui vient de l'écrivain, mais également des maisons d'éditions et des médias, implique cependant toujours une part de risque et d'incertitude, dans la mesure où la perception de la transgression par le public n'est jamais totalement prévisible.

Pour voir si ce paradoxe se retrouve dans Soumission, nous allons d'abord essayer de déceler dans le roman des éléments concrets de transgression, lesquels pourraient donner des indications sur la volonté de l'auteur de provoquer le lecteur et/ou l'autorité.

\section{Un scénario dérangeant}

Avant de nous arrêter sur des passages en particulier, exposons dans ses grandes lignes l'histoire sur laquelle repose Soumission. À la manière d'un roman d'anticipation, 
Houellebecq met en scène dans son livre la montée au pouvoir d'un parti islamique en 2022 et la conversion douce et presqu'insensible d'une population à des valeurs étrangères à une société laïque comme la France. Si le scénario paraît d'emblée peu réaliste, il repose néanmoins sur une certaine vraisemblance. Pour expliquer cette ascension au pouvoir, l'auteur transpose notamment un mécanisme politique que tout lecteur peut avoir à l'esprit : «Le Front Républicain ». Rappelons que celui-ci désignait une consigne de vote venant à la fois de la gauche et de la droite au second tour des élections présidentielles de 2002 pour faire passer Jacques Chirac contre Jean-Marie Le Pen. Dans le roman de Houellebecq, qui se déroule vingt ans plus tard, c'est le parti fictif, «Fraternité Musulmane», et son leader Mohammed Ben Abbes, qui bénéficient d'une coalition politique pour faire barrage au Front National au second tour des élections présidentielles. Les hommes politiques mis en scène restent en grande partie ceux du monde politique actuel, puisque figurent entre autres Marine Le Pen, François Hollande et Manuel Valls.

Il n'y a pas de doutes que le roman entend jouer sur une inquiétude concernant l'immigration et l'intégration, notamment d'une population de confession musulmane. L'expression de cette inquiétude ne constitue cependant pas en soi une transgression, que ce soit dans un roman ou dans d'autres situations de communication, dans la mesure où il n'y a pas d'interdit qui pèse directement sur celle-ci, contrairement à l'inceste ou au racisme par exemple. C'est plutôt la manière dont est abordé le sujet de l'immigration et de l'intégration qui s'écarte du politiquement correct. La dimension sensible du roman se trouve ainsi du côté du discours politique (qui sert de toile de fond dans le récit), puisque l'immigration est présentée non pas comme un problème social sous un angle neutre, mais comme une menace directe pour les valeurs françaises à travers le spectre de la fiction. Ce faisant, le roman met en scène des idées traditionnellement associées au Front National qui, sur l'échiquier politique, sont jugées comme «réactionnaires», voire comme «racistes ». Comme toujours chez Houellebecq, il s'agit cependant non pas d'une prise de position explicite et assumée - le narrateur se sent d'ailleurs «aussi politisé qu'une serviette de toilette » (Houellebecq 2015, 50) - mais d'un jeu avec les frontières du paysage politique français. En revanche, le roman contient une critique de la vie politique en France en la présentant comme dominée par deux camps, un parti de «centre-gauche » et un autre de «centre-droite », qui ont à peu près les mêmes idées et qui sont incapables de s'adapter aux nouveaux défis posés au vingt-et-unième siècle ${ }^{2}$.

Mais qui est la cible réelle de cette provocation ? Selon nous, la réponse est moins le monde politique et les médias - même si ceux-ci font l'objet d'une certaine critique dans le roman - qu'un groupe beaucoup plus large. En mettant les élections au centre du texte et en introduisant l'idée que la population française se soumet par sa propre volonté à une force étrangère, c'est surtout les Français en général qui sont mis en cause. Cette idée est présente dès le titre «Soumission» qui reprend la signification du mot «Islam » et qui renvoie en même temps à une France soumise par l'Islam. L'incapacité de protester et le manque de volonté s'expliquent dans le roman par le fait que la population est profondément individualiste et manipulée par les médias - un comportement qui mène finalement à une sorte de collaboration avec l'ennemi. La résignation des Français est cependant particulièrement visible à travers le parcours du narrateur, lui-même appelé François, qui, dans un premier temps, ne vote pas, puis, dans un second temps, envisage de se convertir à l'Islam par opportunisme, dans la mesure où il voit dans le nouvel ordre politique une

\footnotetext{
${ }^{2}$ Voir Houellebecq 2015, 50-53.
} 
possibilité de poursuivre sa carrière universitaire et de profiter d'un système, la Charia, qui avantage les hommes au détriment des femmes. Si François n'est qu'un exemple dans cette société fictive en pleine transformation, il incarne de manière particulièrement éloquente le détachement de la population quant au devenir de la société française et aux valeurs qui l'ont fait naître.

La résignation des Français est visible dans la manière dont le récit expose l'altérité. Face à l'Autre, incarné par Mohammed Ben Abbes, qui se fait de plus en plus envahissant à la fois dans les symboles et dans les mœurs, détonne le «nous », quasiment absent, comme si tout sentiment d'appartenance à la société et à une identité collective avait disparu. La résistance au nouvel ordre politique vient exclusivement des groupes nationalistes et d'extrémistes de droite, comme les Identitaires et le Front National, qui organisent une grande manifestation, mais à laquelle ne participe pas la majorité des Français. Quant à François, il observe les événements d'un regard distant devant son écran de télévision, comme on peut le voir dans le passage qui suit :

Je coupai le son, mais continuai un moment à regarder l'image. Une immense banderole barrait toute la largeur de l'avenue, portant l'inscription : «Nous sommes le peuple de France ». Sur de nombreux petits panneaux disséminés dans la foule était écrit, plus simplement : «Nous sommes chez nous » - c'était devenu le slogan, à la fois explicite et dénué d'agressivité exagérée, utilisé par les militants nationaux au cours de leur rassemblement. L'orage menaçait toujours; l'énorme nuage était maintenant suspendu, immobile au-dessus du cortège. Au bout de quelques minutes je me lassai, et me replongeai dans En rade. (Houellebecq 2015, 119-120)

Le roman met en effet en scène plusieurs groupes qui se démarquent par une forte identité collective - c'est surtout le cas des groupes politiques mentionnés précédemment, mais également des communautés religieuses, comme les musulmans et les juifs. En revanche, le Français qui se trouve au centre de l'échiquier politique sans appartenance à une communauté spirituelle, est mis hors-jeu. Pour prendre un exemple particulièrement parlant, on peut citer un passage où Myriam, une étudiante d'origine juive qui a une relation avec François, lui demande ce qui va se passer à l'université après les élections.

- Et toi, qu'est-ce que tu vas faire ? Comment tu crois que ça va se passer, à la fac ? Je l'accompagnai sur le pas de la porte; de fait, je me rendais compte que je n'en avais pas la moindre idée ; et je me rendais compte également que je m'en foutais. Je l'embrassai doucement sur les lèvres avant de répondre : «Il n'y a pas d'Israël pour moi. » Une pensée bien pauvre ; mais une pensée exacte. (Houellebecq 2015, 112)

Dans l'allusion du narrateur à l'exil prochain de Myriam, la société française est opposée de manière implicite à la société israélienne, cimentée autour d'une religion et d'une identité forte. Le manque d'attache de François et d'engagement pour son pays prend ainsi une tournure universelle et non plus seulement personnelle : c'est la société française qui, n'ayant plus de cohésion et plus d'avenir à proposer à ses concitoyens, semble s'autodétruire.

Le roman met ainsi le lecteur face à des questions dérangeantes qui pourraient être exprimées de la manière suivante: les valeurs démocratiques de la société française, symbolisée par la laïcité, ne sont-elles pas menacées par la politique migratoire du pays ? L'intégration des musulmans est-elle possible? La provocation mise à l'œuvre dans Soumission a ainsi la nature d'un défi posé, non pas aux autorités elles-mêmes, ni à un lecteur politisé, mais au citoyen français en général. L’angle choisi réduit cependant la portée 
provocatrice du discours ; on pourrait même considérer que la provocation est parfaitement dosée pour plaire à un large public, puisque le lecteur pourrait soit ressentir le frisson plaisant de la remise en question de soi souvent caractéristique de bons livres, soit faire le choix de ne pas croire au scénario et donc de ne pas s'identifier au destin collectif proposé par la fiction.

Ainsi, Soumission, comme nous venons de le voir, n'offre pas de matière convaincante pour un scandale. Ceci vient du contenu du roman, mais également du genre fictionnel qui ne communique pas d'idées au lecteur de façon simple et directe. Avec Soumission, on est loin du roman à thèse. Pour comprendre le bruit engendré par le roman, il faut donc prendre en compte la manière dont Flammarion, l'éditeur de Houellebecq, a préparé la sortie du livre, l'image que l'écrivain projette dans les médias et la façon dont ceux-ci le présente.

\section{Sous le feu des projecteurs}

Que l'auteur le cherche ou non, une transgression ne cause pas automatiquement de scandale; comme nous l'avons souligné, il faut qu'un tiers, principalement les éditeurs, trouve un intérêt à rendre la transgression publique et un public qui veut y prendre part. En littérature, la logique qui prévaut depuis la fin des années 1970, comme le note Olivier Bessard-Banquy, est celle de l'édition industrielle qui n'attend pas seulement de la presse qu'elle donne un «coup de pouce encourageant », mais aussi qu'elle " orchestre savamment débats et controverses autour de ses publications pour réussir à créer l'événement » (BessardBanquy 2012, 53). Dans le cas de Houellebecq, qui bénéficie d'une grande notoriété notamment grâce à sa présence sur les plateaux de télévision et qui a, depuis ses publications précédentes, une image de provocateur ${ }^{3}$, on peut dire que le nom de l'auteur est presque devenu une marque à lui tout seul. Cela est manifeste pour Soumission, livre pour lequel Houellebecq a été invité au Journal du soir de France 2, présenté par David Pujadas, le 6 janvier 2015, la veille de la publication du roman, pour donner une interview longue de neuf minutes, événement extrêmement rare dans le monde littéraire.

Au moment de cette interview, les médias, démarchés par Flammarion, l'éditeur de Houellebecq, ont déjà, depuis un mois, fait un travail considérable de marketing autour du livre. Différentes chroniques dans de grands journaux et dans des magazines littéraires ont ainsi annoncé un livre qui devait faire parler de lui ${ }^{4}$. La preuve que la sortie du roman est parvenue à créer l'événement est l'apparition de Houellebecq au Journal de France 2, que nous avons choisi d'étudier. Celle-ci illustre justement cette stratégie de type industrielle mise en place par les maisons d'édition qui cherchent à occuper l'espace médiatique pour le lancement d'un livre. Pour le Journal de France 2, on peut penser que l'intérêt est double : inviter un écrivain connu du grand public et parler d'un livre que tout le monde attend. Le Journal, comme nous allons le voir à présent, insiste sur la dimension polémique du roman en mettant en avant le geste provocateur de l'écrivain plutôt que le contenu du roman lui-même.

Le choix d'intégrer une séquence vidéo présentant l'avis de trois personnes qui réagissent au roman est à ce titre révélateur. On y trouve deux philosophes (Malek Chebel et Michela Marzano) et un militant antiraciste (Alain Jakubowicz). Ces intervenants ne portent pas de jugement esthétique sur le roman, mais se prononcent sur des questions d'ordre

\footnotetext{
${ }^{3}$ Voir à ce propos l'article « Posture polémique ou polémisation de la posture ? Le cas de Michel Houellebecq » (2012) de Louise Moor.

${ }^{4}$ Citons entres autres deux articles dans France Info, publiés le 5/12/2014 et le 15/12/2014, un article dans le Parisien publié le 5/12/2014 et un article dans Les Inrockuptibles le 15/12/2014.
} 
politique et sur une responsabilité attribuée à l'écrivain. Marzano dénonce ainsi l'image négative de la femme véhiculée par le roman, Jakubowicz considère quant à lui le roman comme un « cadeau pour Marine Le Pen » et Chebel renvoie l'auteur à ses propos sur l'Islam qui ont fait scandale lors de la publication du roman Plateforme en 2001, presque 15 ans auparavant : «La religion la plus con, c'est quand même l'islam. Quand on lit le Coran, on est effondré... effondré !» (Houellebecq 2001), propos qui contribuent également à associer l'écrivain à des idées d'extrême-droite (Meizoz 2003, 4).

Les médias tentent ainsi de «jouer la carte» de la misogynie et surtout celle de l'islamophobie pour faire débat, un choix qui semble nourri par l'ethos préalable de provocateur que l'écrivain a construit dans ses œuvres précédentes et par l'expression d'une opinion ancienne sur le sujet. Le but de cette courte séquence vidéo est de créer un débat, sans pour autant exposer directement l'écrivain à des contradicteurs, et d'intéresser aussi des téléspectateurs qui n'ont pas encore lu le livre. Ceux-ci ne le liront peut-être jamais, non pas parce qu'ils trouveraient le sujet dérangeant, mais tout simplement parce qu'ils ne lisent pas de romans ou qu'ils ne s'intéressent pas à cet auteur.

Il faut cependant souligner que ce qui voudrait être présenté comme «scandaleux » au public, ce n'est pas l'islamophobie supposée du roman, mais qu'elle soit associée, dans des proportions très restreintes, à un écrivain de renom - le statut social du transgresseur étant en effet central pour donner lieu à un scandale (Adut 2008, 21). Pour s'en convaincre, on peut avoir à l'esprit qu'un tel programme, le Journal de France 2, diffusée sur une chaîne du service public, est écouté par des millions de téléspectateurs et ne peut pas se permettre de faire la promotion d'une œuvre islamophobe ou d'un écrivain jugé comme tel.

L'image de provocateur de Houellebecq, établie de longue date auprès d'un large public, apporte donc une garantie de donner un goût de transgression sans risque de dépasser les limites de l'acceptable pour les téléspectateurs. Cependant, en présentant le roman sous l'angle de l'islamophobie, les médias poussent Houellebecq à prendre position, et cela même si, dans le roman, l'opinion de l'auteur reste impossible à déterminer. Face à ces attaques, on peut remarquer que l'écrivain assume la provocation tout en prenant soin de limiter sa portée : « je ne peux pas dire que j'aime [la polémique] mais je ne fais pas non plus l'effort de l'éviter. [...] Il faut essayer de tout traiter ». Cependant, face à l'insistance de Pujadas sur la matière sensible de Soumission, Houellebecq choisit, ou plutôt est contraint de ramener les questions du présentateur au scénario de son roman, préférant souligner le relativisme des propos des personnages et le fait que la fiction est un terrain d'expérimentation pour l'esprit : « il faut pas juger les gens quand on écrit [...] il faut que tous les personnages aient raison ». En effet, tandis que le journaliste cherche à réduire le roman à un message politique provocateur, l'écrivain essaie de mettre l'accent sur des aspects plus littéraires de son roman. Sous les feux de la rampe où le scandale est censé atteindre un comble, se crée au lieu un point d'anticlimax, parce que l'écrivain tente de faire dégonfler la polémique en répondant de manière vague aux questions du journaliste qui lui demande de prendre position sur divers sujets. Citons ici quelques-unes des questions :

- Est-ce que vous aimez ça, finalement, la polémique, parce que vous semblez la rechercher?

- Qu'est-ce que c'est, Michel Houellebecq, c'est de la pure fiction, c'est votre vision de l'avenir?

- Michel Houellebecq, un cadeau de Noël à Marine Le Pen ? 
- Est-ce que vous approuvez votre héros lorsqu'il se convertit?

- Notre culture occidentale, celle de l'individualisme, celle du libéralisme, est-elle si creuse, elle est si finissante, si agonisante que ça?

- En tout cas, on est condamné ?

- Et vous, vous le ressentez personnellement?

Si Houellebecq tente par ses réponses de recentrer la discussion sur le roman, le journaliste lui demande systématiquement d'exprimer des opinions personnelles, plus à même d'intéresser la majorité des téléspectateurs.

L'écart entre le discours du présentateur, intéressé par ce que pense l'écrivain, et celui du romancier, qui tente de parler du contenu de son roman, fait ressortir le côté fabriqué du scandale annoncé et crée une situation quelque peu paradoxale : alors que le roman n'est pas encore sorti, le journaliste insinue par ses questions une polémique et des réactions qui n'ont pas encore eu lieu chez le public. C'est une tendance qui affecte potentiellement la formation des scandales littéraires et leur perception, et qui tend à les réduire à un simulacre lié à la promotion des livres.

On ne peut pas dire pour autant que l'écrivain soit victime de ce procédé médiatique, dans la mesure où il en bénéficie pour faire parler de son livre et pour médiatiser son image. En effet, comme l'explique Bessard-Banquy, la présence à la télévision d'un écrivain implique une consécration à l'époque actuelle des médias de masse :

Le passage sur le plateau miraculeux, loin d'être une simple récompense pour l'auteur, s'avère être une sorte de conversion symbolique, garantissant à l'heureux élu une accession soudaine au firmament des grands auteurs (aux yeux du public) quand cinquante ans plus tôt seule une publication chez Gallimard pouvait avoir une valeur équivalente. (Bessard-Banquy 2012, 52)

Dans le contexte qui nous concerne, la collaboration entre le journaliste et l'écrivain se fait naturellement dans une logique de gagnant-gagnant. Cependant, pour que le bruit engendré par le roman ne soit pas préjudiciable pour son image, l'écrivain doit veiller à ne pas être catalogué comme auteur d'extrême-droite, sans pour autant renoncer à rester un écrivain provocateur. De plus, en pouvant apparaître comme un auteur qui cherche un succès de scandale facile, Houellebecq court le risque de ne pas bénéficier du soutien de la presse spécialisée et des critiques littéraires, nécessaires pour entretenir sa crédibilité. La teneur polémique introduite par les médias offre ainsi de la publicité au roman et à son auteur mais ne garantit pas pour autant un effet positif sur son image d'auteur - c'est la réception critique qui est déterminante et qui dira si le roman est jugé réussi. L'écrivain doit donc jouer sur deux tableaux: les médias et les spécialistes. Son apparition médiatique témoigne de ce jeu d'équilibre fragile, d'autant que, comme nous l'avons vu, même une émission sérieuse comme le journal de France 2, choisit de jouer avec le thème de l'islamophobie au lieu de s'intéresser à la dimension littéraire du roman.

On notera que le coup médiatique semble avoir été une réussite pour le Journal de France 2, qui a pu avoir l'exclusivité de parler de Soumission à la télévision avant sa diffusion en librairie, ainsi que pour Houellebecq, car suite à la sortie du roman, la polémique, qui n'a pas fait long feu, a néanmoins été suffisante pour éveiller l'intérêt du public. Les critiques venant de la presse écrite ont rapidement nuancé le jugement premier du roman, en mettant en 
avant l'absence de programme politique et de prise de position islamophobe. Une partie des critiques non négligeables a par ailleurs défendue les qualités littéraires du roman, comme on peut le voir dans ces extraits d'articles publiés dans Libération à la sortie du roman.

Soumission est un roman délibérément piégé, qui exige une lecture critique et attentive parce qu'il repose sur un processus constant de réversibilité. (Novak-Lechevalier 2015)

Son style est là : neutralité féroce, phrases nettes, coups de pattes, sens du dialogue et de l'absurde, dégagements philosophiques, italiques à l'ironie sociologique, points virgule à la presque Flaubert. (Lançon 2015)

Houellebecq est un écrivain, un vrai. (Joffrin 2015)

\section{Conclusion}

L'étude des aspects polémiques de Soumission et leur amplification dans une émission de grande écoute a permis de faire apparaître que le bruit médiatique autour de Soumission de Houellebecq se caractérise par un côté construit et préparé, qui le distingue du fracas imprévu et incontrôlé des scandales sous leur forme traditionnelle. Si comme nous l'avons dit, c'est le propre des maisons d'édition d'essayer de créer l'événement en se servant des médias, on a également pu voir que ces derniers, loin d'être au service des éditeurs et des écrivains, suivent leur propre logique. L'intérêt de ces médias n'est pas nécessairement la vente ; l'invitation d'un écrivain de renom ajoute une touche « culturelle » valorisante à un programme comme le journal télévisé, et une pointe de frisson bienvenue si celui-ci a en plus une image de provocateur comme Houellebecq. Une conséquence de ce rôle central des médias dans le modèle industriel de l'édition est le contrôle indirect qu'ils exercent sur la littérature en imposant une manière de la diffuser, voire de simplifier son contenu, pour en présenter certains aspects sous un jour susceptible d'intéresser les téléspectateurs - dont une grande partie n'est pas lecteurs de romans.

Le passage de Houellebecq au Journal de France 2, montre que les médias peuvent aujourd'hui intervenir à un stade beaucoup plus précoce dans l'apparition d'un scandale littéraire que ne le montre le schéma présenté dans la partie théorique, lequel est donc sujet à quelques modifications. Dans une logique de montée aux médias, l'écrivain rend aujourd'hui publique sa transgression non seulement par l'intermédiaire de l'éditeur, mais aussi directement par les médias. Ceci a potentiellement des effets importants sur la perception de l'événement d'une part, et, d'autre part, sur les choix littéraires même de l'écrivain qui devra assumer ce qu'il a écrit sur des plateaux de télévision ou de radio. En effet, si c'est l'éditeur qui prend contact avec les médias et non pas l'écrivain, c'est ce dernier qui donne les interviewes et qui se met en scène selon ses propres choix. Cette circonstance explique notre choix de faire figurer un lien direct entre l'écrivain et les médias dans ce que nous pourrions appeler une «montée aux médias par la provocation» (Fig. 2). Dans ce nouveau schéma, nous avons également jugé utile de faire apparaître deux publics: les lecteurs du livre confrontés à la fiction et les téléspectateurs confrontés à une interprétation du roman conçue par les journalistes. Ces publics ne sont naturellement pas tout à fait distincts, mais sont susceptibles de se superposer. 


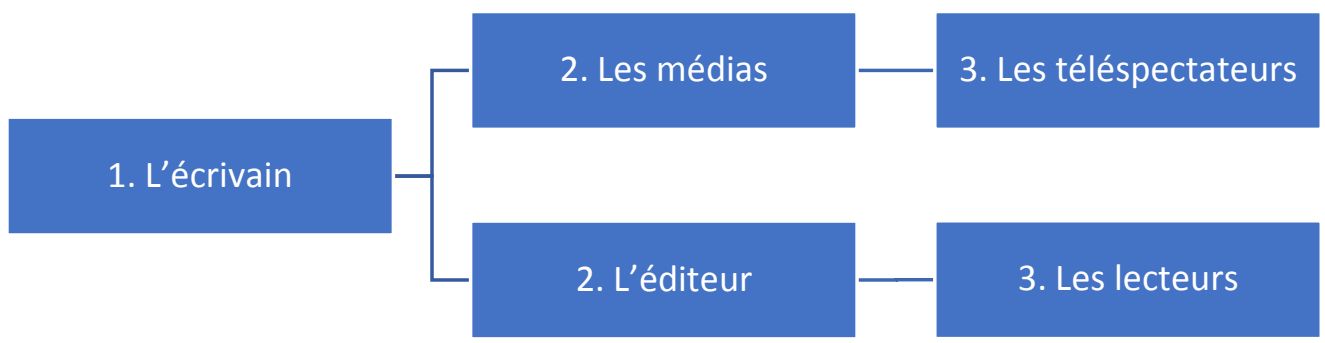

Fig. 2 Montée aux médias par la provocation

L'exemple de Soumission ouvre par ailleurs pour la recherche sur les scandales littéraires de nouvelles perspectives de questionnement. On peut par exemple constater que, si les médias offrent une caisse de résonnance pour les scandales littéraires à notre époque, se pose alors le problème du lien de dépendance qui se crée entre les écrivains et les médias, lesquels risquent d'interférer fortement sur l'autonomie de la littérature, déjà sujette à des contraintes comme nous l'avons vu. Dès lors c'est la capacité même de la littérature à être réellement transgressive qui est remise en cause, tout du moins de celle qui mise sur les médias.

\section{Bibliographie}

Adut, Ari. 2008. On scandal. Moral Disturbances in Society, Politics and Art. Cambridge: Cambridge University Press.

Angenot, Marc. 1982. La Parole pamphlétaire: contribution à la typologie des discours modernes. Paris: Payot.

Amossy, Ruth. 2014. Apologie de la polémique. Paris: Presses universitaires de France.

Bessard-Banquy, Olivier. 2012. L'industrie des lettres: étude sur l'édition littéraire contemporaine. Paris: Pocket.

Estier, Samuel. 2015. À propos du «style» de Houellebecq: retour sur une controverse (1998-2011). Lausanne: Archipel essais.

Houellebecq, Michel. 2001. «Michel Houellebecq». Interview by Didier Sénécal. Lire, September 1, 2001.

https://www.lexpress.fr/culture/livre/michelhouellebecq_804761.html\#EwmlBKQz13 1KbYyp.99

Houellebecq, Michel. 2015. «Journal du soir». Interview by David Pujadas, France 2, January 6, 2015. https://www.youtube.com/watch?v=Nx7OPRmpkmM

Houellebecq, Michel. 2015. Soumission. Paris: Flammarion.

Joffrin, Laurent. «"Soumission". Le Pen au Flore». Libération, January 2, 2015. http://next.liberation.fr/livres/2015/01/02/le-pen-au-flore_1173182

Lançon, Philippe. 2015. «Houellebecq et le Coran ascendant». Libération, January 2, 2015. http://next.liberation.fr/livres/2015/01/02/houellebecq-et-le-coran-ascendant_1173203

Meizoz, Jérôme. 2003. «Le roman et l'inacceptable: polémiques autour de Plateforme de Michel Houllebecq. » Études de Lettres, no. 266. «Littérature et morale publique : censure, justice, presse, XVIIe-XXe siècles ». 
Moor, Louise. 2012. «Posture polémique ou polémisation de la posture ? », COnTEXTES, no. 10. http://journals.openedition.org/contextes/4921

Novak-Lechevalier, Agathe. 2015. " "Soumission". La littérature comme résistance». Libération. March 1, 2015. http://next.liberation.fr/culture/2015/03/01/soumission-lalitterature-comme-resistance 1212088

Philippe, Gilles, 2004. Flaubert savait-il écrire ?: une querelle grammaticale, 1919-1921. Grenoble: ELLUG, Université Stendhal. 